PROCEEDINGS OF THE

AMERICAN MATHEMATICAL SOCIETY

Volume 132, Number 11, Pages 3357-3365

S 0002-9939(04)07580-X

Article electronically published on June 21, 2004

\title{
ON TWO PROBLEMS OF ERDÖS AND HECHLER: NEW METHODS IN SINGULAR MADNESS
}

\author{
MENACHEM KOJMAN, WIESŁAW KUBIŚ, AND SAHARON SHELAH
}

(Communicated by Carl G. Jockusch, Jr.)

\begin{abstract}
For an infinite cardinal $\mu, \operatorname{MAD}(\mu)$ denotes the set of all cardinalities of nontrivial maximal almost disjoint families over $\mu$.

Erdős and Hechler proved in 1973 the consistency of $\mu \in \operatorname{MAD}(\mu)$ for a singular cardinal $\mu$ and asked if it was ever possible for a singular $\mu$ that $\mu \notin \operatorname{MAD}(\mu)$, and also whether $2^{\text {cf } \mu}<\mu \Longrightarrow \mu \in \operatorname{MAD}(\mu)$ for every singular cardinal $\mu$.

We introduce a new method for controlling $\operatorname{MAD}(\mu)$ for a singular $\mu$ and, among other new results about the structure of $\operatorname{MAD}(\mu)$ for singular $\mu$, settle both problems affirmatively.
\end{abstract}

\section{INTRODUCTION}

1.1. Background. Let $\mu$ be an infinite cardinal. A family of sets $\mathcal{A}$ is $\mu$-almost disjoint ( $\mu$-ad for short) if $|A|=\mu=|\bigcup \mathcal{A}|$ for every $A \in \mathcal{A}$ and $|A \cap B|<\mu$ for every distinct $A, B \in \mathcal{A}$. $\mathcal{A}$ is maximal $\mu$-almost disjoint ( $\mu$-mad) if there is no $C \subseteq \cup \mathcal{A}$ such that $\mathcal{A} \cup\{C\}$ is $\mu$-almost disjoint; in this case we also say that $\mathcal{A}$ is mad in $\mu$. It is clear that every $\mu$-almost disjoint family consisting of fewer than cf $\mu$ sets is mad in $\mu$; such a family will be called trivial. We denote by $\operatorname{MAD}(\mu)$ the set of all cardinalities of nontrivial mad families in $\mu$. A standard diagonalization argument shows that ef $\mu \notin \operatorname{MAD}(\mu)$. Therefore, $\operatorname{MAD}(\mu)$ is contained in the interval of cardinals $\left[\operatorname{cf}(\mu)^{+}, 2^{\mu}\right]$.

W. W. Comfort asked (see [7]) under what conditions it follows that $\mu \in \operatorname{MAD}(\mu)$ for a singular cardinal $\mu$. P. Erdős and S. Hechler [7] proved that $\mu \in \operatorname{MAD}(\mu)$ if $\lambda^{\text {cf } \mu}<\mu$ for every $\lambda<\mu$. Thus, if $2^{\aleph_{0}}<\aleph_{\omega}$, then the interval $\left[2^{\aleph_{0}}, \aleph_{\omega}\right]$ of cardinals is contained in $\operatorname{MAD}\left(\aleph_{\omega}\right)$.

Erdös and Hechler asked in [7] whether it is consistent that $\mu \notin \operatorname{MAD}(\mu)$ for some singular cardinal $\mu$ and, more concretely, whether Martin's axiom together with $2^{\aleph_{0}}>\aleph_{\omega}$ implies that $\aleph_{\omega} \notin \operatorname{MAD}\left(\aleph_{\omega}\right)$. They also asked whether $2^{\text {cf } \mu}<\mu$ implies $\mu \in \operatorname{MAD}(\mu)$ for singular cardinals $\mu$ other than $\aleph_{\omega}$.

Received by the editors June 10, 2002 and, in revised form, September 10, 2002.

2000 Mathematics Subject Classification. Primary 03E10, 03E04, 03E17, 03E35; Secondary 03E55, 03E50.

Key words and phrases. Almost disjoint family, singular cardinal, bounding number, smooth pcf scales.

The first author's research partially supported by an Israeli Science Foundation grant no. $177 / 01$.

The third author's research was supported by The Israel Science Foundation, Publication 793.

(C)2004 American Mathematical Society 
Both problems are settled affirmatively by the general results below on $\operatorname{MAD}(\mu)$ for a singular $\mu$.

1.2. Notation. Let $\mathfrak{a}_{\mu}=\min \operatorname{MAD}(\mu)$, and let $\mathfrak{a}=\mathfrak{a}_{\aleph_{0}}$. For a singular $\mu$ it follows that $\operatorname{MAD}(\operatorname{cf} \mu) \subseteq \operatorname{MAD}(\mu)$; therefore $\mathfrak{a}_{\mu} \leqslant \mathfrak{a}_{\text {cf } \mu}$.

A crucial role in the results is played by two bounding numbers: $\mathfrak{b}_{\mu}$ and $\mathfrak{b}_{\text {cf } \mu}$.

For every quasi-ordering $(P, \leqslant)$ with no maximum, the bounding number $\mathfrak{b}(P, \leqslant)$ is the least cardinality of a subset of $P$ with no upper bound. For a regular cardinal $\kappa$, let $\mathfrak{b}_{\kappa}$ denote the bounding number of $\left(\kappa^{\kappa}, \leqslant^{*}\right)$, where $f \leqslant * g$ means that $\mid\{i<$ $\kappa: f(i)>g(i)\} \mid<\kappa ;$ let $\mathfrak{b}=\mathfrak{b}_{\aleph_{0}}$. It is well known that $\kappa<\mathfrak{b}_{\kappa} \leqslant \mathfrak{a}_{\kappa}$ for a regular cardinal $\kappa$ (for $\kappa=\aleph_{0}$ see [6]; the general case is similar) and that under Martin's axiom, $\mathfrak{b}=2^{\aleph_{0}}$.

Suppose that $\mu$ is a singular cardinal of cofinality $\kappa$ and that $\left\langle\mu_{i}: i<\kappa\right\rangle$ is a strictly increasing sequence of regular cardinals with supremum $\mu$. Standard diagonalization shows that $\mathfrak{b}\left(\prod_{i<\kappa} \mu_{i}, \leqslant^{*}\right)>\mu$. Denote by $\mathfrak{b}_{\mu}$ the supremum of $\mathfrak{b}\left(\prod \mu_{i}, \leqslant^{*}\right)$ over all strictly increasing sequences of regular cardinals $\left\langle\mu_{i}: i<\kappa\right\rangle$ with supremum $\mu$.

Each of the following three relations is consistent with ZFC: $\mathfrak{b}<\mathfrak{b}_{\aleph_{\omega}}, \mathfrak{b}=\mathfrak{b}_{\aleph_{\omega}}$ and $\mathfrak{b}>\mathfrak{b}_{\aleph_{\omega}}$.

1.3. The results. We prove that for every singular cardinal $\mu$ :

(1) $\mathfrak{a}_{\mu} \geqslant \min \left\{\mathfrak{b}_{\mu}, \mathfrak{b}_{\operatorname{cf}} \mu\right.$;

(2) $\mathfrak{a}_{\mu} \leqslant \lambda<\mathfrak{b}_{\mu} \Longrightarrow \lambda \in \operatorname{MAD}(\mu)$.

Thus, if $\mathfrak{b}_{\text {cf } \mu}>\mu$, it follows from (1) that $\mathfrak{a}_{\mu}>\mu$, and hence $\mu \notin \operatorname{MAD}(\mu)$; and if $\mathfrak{a}_{\mathrm{cf}} \mu<\mu$, it follows from (2) that $\mu \in \operatorname{MAD}(\mu)$. In particular,

(b) $M A+2^{\aleph_{0}}>\aleph_{\omega} \Longrightarrow \aleph_{\omega} \notin \operatorname{MAD}\left(\aleph_{\omega}\right)$,

(a) $2^{\text {cf } \mu}<\mu \Longrightarrow \mu \in \operatorname{MAD}(\mu)$ for every singular $\mu$,

which, respectively, settle in the affirmative both problems of Erdős and Hechler from [7].

If one assumes the consistency of large cardinals, $\mathfrak{b}_{\aleph_{\omega}}$ can be shifted up arbitrarily high below $\aleph_{\omega_{1}}$. Following this with a ccc forcing for controlling $\mathfrak{b}$ proves the following:

(3) for every regular $\lambda \in\left(\aleph_{\omega}, \aleph_{\omega_{1}}\right)$ and regular uncountable $\theta \leqslant \lambda^{+}$, it is consistent that

$$
\operatorname{MAD}\left(\aleph_{\omega}\right)=\left[\theta, \lambda^{+}\right] .
$$

So, e.g., the following are consistent:

- $\operatorname{MAD}\left(\aleph_{\omega}\right)=\left\{\aleph_{1}, \aleph_{2}, \ldots, \aleph_{\omega+\beta+2}=2^{\aleph_{\omega}}\right\}$ for an arbitrary $\beta<\omega_{1}$,

- $\operatorname{MAD}\left(\aleph_{\omega}\right)=\left\{\aleph_{\omega+\beta+2}\right\}$ for an arbitrary $\beta<\omega_{1}$,

- $\operatorname{MAD}\left(\aleph_{\omega}\right)=\left[\aleph_{\omega+\alpha+1}, \aleph_{\omega+\beta+2}\right]$ for arbitrary $\alpha \leqslant \beta<\omega_{1}$,

and so on.

We refer the reader to the comprehensive list of references in D. Monk's recent paper [12, in which maximal almost disjoint families are viewed as partitions of unity in the Boolean algebra $\mathcal{P}(\mu){ }_{[\mu]}<\mu$.

1.4. Preliminary facts. We will use the following facts from [7]:

(1) $\operatorname{MAD}(\operatorname{cf} \mu) \subseteq \operatorname{MAD}(\mu)$, and

(2) $\operatorname{MAD}(\mu)$ is closed under singular suprema.

The latter fact is stated in [7] in a less general form. So we give a proof here. 
Lemma 1.1. Assume that $\lambda=\sup _{i<\theta} \lambda_{i}$, where $\left\{\lambda_{i}: i<\theta\right\} \subseteq \operatorname{MAD}(\mu)$ and $\theta<\lambda$. Then $\lambda \in \operatorname{MAD}(\mu)$.

Proof. We may assume that $\theta \leqslant \lambda_{0}$. Let $\mathcal{A}$ be a mad family in $\mu$ with $|\mathcal{A}|=\lambda_{0}$. Write $\mathcal{A}=\left\{A_{i}: i<\lambda_{0}\right\}$ and for each $i<\theta$ choose a mad family $\mathcal{B}_{i}$ with $\bigcup \mathcal{B}_{i}=A_{i}$ and $\left|\mathcal{B}_{i}\right|=\lambda_{i}$. Set

$$
\mathcal{C}=\bigcup_{i<\theta} \mathcal{B}_{i} \cup\left\{A_{j}: \theta \leqslant j<\lambda_{0}\right\} .
$$

Then $|\mathcal{C}|=\lambda$ and $\mathcal{C}$ is $\operatorname{mad}$ in $\mu$.

The following fact will also be used in some proofs.

Lemma 1.2. Let $\kappa=\operatorname{cf} \mu$, and let $\mathcal{A}$ be a $\mu$-almost disjoint family of size $\kappa$. Then there exists a mad family $\mathcal{A}^{\prime} \supseteq \mathcal{A}$ such that $\left|\mathcal{A}^{\prime}\right|=\mathfrak{a}_{\mu}$ and $\bigcup \mathcal{A}^{\prime}=\bigcup \mathcal{A}$.

Proof. Fix a $\mu$-mad family $\mathcal{B}$ with $|\mathcal{B}|=\mathfrak{a}_{\mu}$. Choose $\mathcal{B}_{0}=\left\{B_{i}: i<\kappa\right\} \subseteq \mathcal{B}$. Let $X=\bigcup_{i<\kappa} B_{i}$ and define

$$
\mathcal{B}^{\prime}=\left\{B \cap X: X \in \mathcal{B} \backslash \mathcal{B}_{0} \&|X \cap B|=\mu\right\} .
$$

Let $\left\langle A_{i}: i<\kappa\right\rangle$ be a one-to-one enumeration of $\mathcal{A}$. Define a bijection $f: \cup \mathcal{A} \rightarrow X$ so that $f\left[A_{i} \backslash \bigcup_{j<i} A_{j}\right]=B_{i} \backslash \bigcup_{j<i} B_{j}$. Finally, set $\mathcal{A}^{\prime}=\mathcal{A} \cup\left\{f^{-1}[B]: B \in \mathcal{B}^{\prime}\right\}$. Observe that $\mathcal{A}^{\prime}$ is mad and $\left|\mathcal{A}^{\prime}\right|=\mathfrak{a}_{\mu}$.

\section{INEQUALITIES}

From now on, $\mu$ will always denote a singular cardinal whose cofinality is denoted by $\kappa$.

\subsection{Bounding numbers and madness in singular cardinals.}

Theorem 2.1. For every singular cardinal $\mu$,

$$
\mathfrak{a}_{\mu} \geqslant \min \left\{\mathfrak{b}_{\mu}, \mathfrak{b}_{\text {cf } \mu}\right\}
$$

Proof. Let $\kappa=\operatorname{cf} \mu$. Suppose to the contrary that $\mathfrak{a}_{\mu}<\min \left\{\mathfrak{b}_{\mu}, \mathfrak{b}_{\kappa}\right\}$, and fix a strictly increasing sequence of regular cardinals $\left\langle\mu_{i}: i\langle\kappa\rangle\right.$ with supremum $\mu$ such that $\mathfrak{b}\left(\prod_{i<\kappa} \mu_{i}, \leqslant^{*}\right)>\mathfrak{a}_{\mu}$.

Let $\mathcal{A}=\{\{i\} \times \mu: i<\kappa\}$. By Lemma 1.2, there exists a family $\mathcal{B} \subseteq[\kappa \times \mu]^{\mu}$ such that $\mathcal{B} \cup \mathcal{A}$ is $\operatorname{mad}$ in $\mu, \mathcal{B} \cap \mathcal{A}=\emptyset$ and $|\mathcal{B}|=\mathfrak{a}_{\mu}$.

For each $B \in \mathcal{B}$, define a function $f_{B}: \kappa \rightarrow \kappa$ by $f_{B}(i)=\min \{j<\kappa: \mid B \cap(\{i\} \times$ $\left.\mu) \mid<\mu_{j}\right\}$. This function is well defined, since $|B \cap(\{i\} \times \mu)|<\mu$ for each $i<\kappa$.

Since $|\mathcal{B}|=\mathfrak{a}_{\mu}<\mathfrak{b}_{\kappa}$, there exists a function $f: \kappa \rightarrow \kappa$ so that $f_{B}<^{*} f$ for all $B \in \mathcal{B}$. Without loss of generality, we may assume that $f$ is strictly increasing.

For each $B \in \mathcal{B}$, for all but boundedly many $i<\kappa$, it follows that $\sup \{\alpha<$ $\left.\mu_{f(i)}:(i, \alpha) \in B\right\}<\mu_{f(i)}$. Let $g_{B}(i)$ be defined by

$$
g_{B}(i)= \begin{cases}0 & \text { if } \sup \left\{\alpha<\mu_{f(i)}:(i, \alpha) \in B\right\}=\mu_{f(i)}, \\ \sup \left\{\alpha<\mu_{f(i)}:(i, \alpha) \in B\right\} & \text { otherwise. }\end{cases}
$$

For each $B \in \mathcal{B}$ the function $g_{B}$ belongs to $\prod_{i<\kappa} \mu_{f(i)}$. Since

$$
\mathfrak{b}\left(\prod_{i<\kappa} \mu_{f(i)}, \leqslant^{*}\right) \geqslant \mathfrak{b}\left(\prod_{i<\kappa} \mu_{i}\right)>\mathfrak{a}_{\mu}
$$

we can fix a function $g \in \prod_{i<\kappa} \mu_{f(i)}$ so that $g_{B}<^{*} g$ for all $B \in \mathcal{B}$. 
Define

$$
C=\bigcup_{i<\kappa}\{i\} \times\left[g(i), \mu_{f(i)}\right) .
$$

Clearly, $|C|=\mu$. For each $B \in \mathcal{B}$ there exists $j_{B}<\kappa$ such that $g_{B}(i)<g(i)$ for all $i>j_{B}$. This implies that $\{i\} \times\left[g(i), \mu_{f(i)}\right)$ is disjoint from $B$ for all $i>j_{B}$. Hence $|B \cap C| \leqslant \mu_{f\left(j_{B}\right)}<\mu$. Clearly, $|C \cap(\{i\} \times \mu)| \leqslant \mu_{f(i)}<\mu$ for all $i<\kappa$; so $\mathcal{A} \cup \mathcal{B} \cup\{C\}$ is $\mu$-almost disjoint, contrary to the maximality of $\mathcal{A} \cup \mathcal{B}$.

A positive answer to the first question of Comfort, Erdős and Hechler follows now as a corollary:

Corollary 2.2. If Martin's Axiom holds and $2^{\aleph_{0}}>\mu>\operatorname{cf} \mu=\aleph_{0}$, then $\mu \notin$ $\operatorname{MAD}(\mu)$.

2.2. Between $\mathfrak{a}_{\mu}$ and $\mathfrak{b}_{\mu}$. In this section we shall show that $\operatorname{MAD}(\mu)$ contains the interval of cardinals $\left[\mathfrak{a}_{\mu}, \mathfrak{b}_{\mu}\right)$ and even $\left[\mathfrak{a}_{\mu}, \mathfrak{b}_{\mu}\right]$ in the case that $\mathfrak{b}_{\mu}$ is a successor of a regular cardinal.

Theorem 2.3. For every singular cardinal $\mu$ and every cardinal $\lambda$,

$$
\mathfrak{a}_{\mu} \leqslant \lambda<\mathfrak{b}_{\mu} \Longrightarrow \lambda \in \operatorname{MAD}(\mu)
$$

If $\mathfrak{b}_{\mu}$ is a successor of a regular cardinal, then $\mathfrak{a}_{\mu} \leqslant \mathfrak{b}_{\mu} \Longrightarrow \mathfrak{b}_{\mu} \in \operatorname{MAD}(\mu)$.

To prove the theorem it suffices, by Lemma 1.1 to show that every regular $\lambda \in\left[\mathfrak{a}_{\mu}, \mathfrak{b}_{\mu}\right)$ belongs to $\operatorname{MAD}(\mu)$.

The proof of this will now be divided into two cases. First we prove that every regular $\mathfrak{a}_{\mu}<\lambda<\mu$ belongs to $\operatorname{MAD}(\mu)$. The proof in this case does not require any specialized techniques. Then we prove the same for regular $\mu<\lambda<\mathfrak{b}_{\mu}$ and for $\mathfrak{b}_{\mu}$ itself when it is the successor of a regular cardinal. In this case the proof requires some machinery from pcf theory.

Despite the technical differences between both proofs, they are similar, and could, in fact, be combined to a single proof. Both follow the same scheme of gluing together $\lambda$ different $\mu$-mad families, each of size $\mathfrak{a}_{\mu}$, to a single $\mu$-mad family of size $\lambda$. In the case $\lambda<\mu$, a simple presentation of $\mu$ as a disjoint union of $\lambda$ parts works; in the second part we need to rely on smooth pcf scales to get a presentation of $\mu$ as an almost increasing and continuous union of length $\lambda$ of sets of size $\mu$.

2.2.1. The case $\lambda<\mu$.

Lemma 2.4. Suppose $\mu>\operatorname{cf} \mu=\kappa$. Then for every regular cardinal $\lambda$,

$$
\mathfrak{a}_{\mu} \leqslant \lambda<\mu \Longrightarrow \lambda \in \operatorname{MAD}(\mu) .
$$

Proof. Suppose $\lambda$ is regular and $\mathfrak{a}_{\mu} \leqslant \lambda<\mu$. Since $\mathfrak{a}_{\mu}>\kappa=\operatorname{cf} \mu, \lambda>\kappa$.

Fix a strictly increasing sequence of regular cardinals $\left\langle\mu_{i}: i<\kappa\right\rangle$ such that $\sup _{i<\kappa} \mu_{i}=\mu$ and $\lambda<\mu_{0}$. We will work in $\mu \times \lambda$ instead of $\mu$. Let $S=\{\delta<$ $\lambda$ : $\operatorname{cf} \delta=\kappa\}$. For each $\delta \in S$ fix a strictly increasing, continuous sequence $D_{\delta}=$ $\left\langle\gamma_{i}^{\delta}: i<\kappa\right\rangle$ with limit $\delta$ such that $\gamma_{0}^{\delta}=0$. Define

$$
F_{j}^{\delta}=\bigcup\left\{\mu \times\{\beta\}: \gamma_{j}^{\delta} \leqslant \beta<\gamma_{j+1}^{\delta}\right\} .
$$

Thus $\mathcal{F}_{\delta}=\left\{F_{j}^{\delta}: j<\kappa\right\}$ is a disjoint family of sets, each set of size $\mu$, which covers $\mu \times \delta$. Let $\mathcal{A}_{\delta} \subseteq[\mu \times \delta]^{\mu}$ be such that $\mathcal{A}_{\delta} \cup \mathcal{F}_{\delta}$ is $\operatorname{mad}$ in $\mu \times \delta, \mathcal{A}_{\delta} \cap \mathcal{F}_{\delta}=\emptyset$ and $\left|\mathcal{A}_{\delta}\right|=\mathfrak{a}_{\mu}$. 
Define

$$
\mathcal{B}=\{\mu \times\{\alpha\}: \alpha<\lambda\} \cup \bigcup_{\delta \in S} \mathcal{A}_{\delta} .
$$

Then $|\mathcal{B}|=\lambda$ and $\mathcal{B} \subseteq[\mu \times \lambda]^{\mu}$. We will show that $\mathcal{B}$ is $\mu$-mad.

First, observe that $\mathcal{B}$ is almost disjoint: clearly each element of $\mathcal{A}_{\delta}$ is almost disjoint from any set of the form $\mu \times\{\alpha\}$, because if $\alpha<\delta$, then $\mu \times\{\alpha\} \subseteq F_{j}^{\delta}$ for $j<\kappa$ such that $\gamma_{j}^{\delta} \leqslant \alpha<\gamma_{j+1}^{\delta}$. Finally, consider $A_{i} \in \mathcal{A}_{\delta_{i}}, i<2$, with $\delta_{0}<\delta_{1}$. Then $A_{0} \subseteq \bigcup_{j<j_{0}} F_{j}^{\delta_{1}}$, where $j_{0}<\kappa$ is such that $\delta_{0}<\gamma_{j_{0}}^{\delta_{1}}$. Thus $\left|A_{0} \cap A_{1}\right|<\mu$.

To see that $\mathcal{B}$ is mad, fix an arbitrary $Z \in[\mu \times \lambda]^{\mu}$. There exists a sequence $\left\langle\alpha_{i}: i<\kappa\right\rangle$ in $\lambda$ such that

$$
\left|Z \cap\left(\mu \times\left\{\alpha_{i}\right\}\right)\right| \geqslant \mu_{i}
$$

If $\left|\left\{\alpha_{i}: i<\kappa\right\}\right|<\kappa$, then $|Z \cap(\mu \times\{\alpha\})|=\mu$ for some $\alpha$. So suppose that $\left|Z \cap\left(\mu \times\left\{\alpha_{i}\right\}\right)\right|<\mu$ for every $i<\kappa$. Taking a subsequence, we may assume that $\left\langle\alpha_{i}: i<\kappa\right\rangle$ is strictly increasing. Let $\delta$ be its supremum. By regularity of $\lambda, \delta \in S$ and therefore $Z \in[\mu \times \delta]^{\mu}$. Shrinking $Z$ if necessary, assume that $Z \subseteq \bigcup_{i<\kappa} \mu \times\left\{\alpha_{i}\right\}$. Then $\left|Z \cap F_{j}^{\delta}\right|<\mu$ for every $j<\kappa$. Thus, $|Z \cap A|=\mu$ for some $A \in \mathcal{A}_{\delta}$. This completes the proof.

Corollary 2.5. Let $\mu>$ cf $\mu=\kappa$. If $\mathfrak{a}_{\kappa} \leqslant \mu$, then $\left[\mathfrak{a}_{\kappa}, \mu\right] \subseteq \operatorname{MAD}(\mu)$. In particular, if $2^{\kappa}<\mu$, then $\mu \in \operatorname{MAD}(\mu)$.

Corollary 2.5 anwers affirmatively the second question of Erdős and Hechler in 7].

2.2.2. The case $\lambda>\mu$. A $(\mu, \lambda)$-scale, for a regular cardinal $\lambda>\mu$, is a sequence $\bar{f}=\left\langle f_{\alpha}: \alpha<\lambda\right\rangle \subseteq \prod_{i<\kappa} \mu_{i}$ such that $\left\langle\mu_{i}: i<\kappa\right\rangle$ is a strictly increasing sequence of regular cardinals with limit $\mu$, and so that $\alpha<\beta<\lambda \Longrightarrow f_{\alpha}<^{*} f_{\beta}$ and for every $g \in \prod_{i<\kappa} \mu_{i}$ there is $\alpha<\lambda$ with $g<^{*} f_{\alpha}$. The relation $f<^{*} g$ means that the set $\{i<\kappa: f(i) \geqslant g(i)\}$ is bounded in $\kappa$. When $\mu$ is fixed, " $(\mu, \lambda)$-scale" will be abbreviated by " $\lambda$-scale". A $\lambda$-scale $\bar{f}$ is smooth if for every $\delta<\lambda$ with $\operatorname{cf} \delta>\kappa$, the sequence $\bar{f} \uparrow \delta=\left\langle f_{\alpha}: \alpha<\delta\right\rangle$ is cofinal in $\left(\prod_{i<\kappa} f_{\delta}(i),<^{*}\right)$. In this case we say that $f_{\delta}$ is an exact upper bound of $\bar{f} \uparrow \delta$. We will denote by $[f, g)$ the set $\{(i, \alpha): i<\kappa \wedge f(i) \leqslant \alpha<g(i)\}$.

The proof in the present case goes through two steps. First, it is shown that whenever a smooth $(\mu, \lambda)$-scale exists and $\mathfrak{a}_{\mu}<\lambda$, it follows that $\lambda \in \operatorname{MAD}(\mu)$. Then it is shown that for every $\mu<\lambda<\mathfrak{b}_{\mu}$ there is a smooth $(\mu, \lambda)$-scale and that in case $\mathfrak{b}_{\mu}$ is a successor of a regular cardinal there is also a smooth $\left(\mu, \mathfrak{b}_{\mu}\right)$-scale.

Lemma 2.6. Assume $\lambda>\mu>$ cf $\mu=\kappa$ and there exists a smooth $(\mu, \lambda)$-scale. If $\mathfrak{a}_{\mu} \leqslant \lambda$, then $\lambda \in \operatorname{MAD}(\mu)$.

Proof. Suppose there exists a smooth $\lambda$-scale $\left\langle g_{\xi}: \xi<\lambda\right\rangle \subseteq \prod_{i<\kappa} \mu_{i}$. Let $S=\{\delta<$ $\lambda$ : $\operatorname{cf} \delta=\kappa\}$, and for each $\delta \in S$ fix a strictly increasing, continuous, sequence $\left\langle\gamma_{i}^{\delta}: i<\kappa\right\rangle$ with limit $\delta$ such that $\gamma_{0}^{\delta}=0$ and put $D_{\delta}=\left\{\gamma_{i}^{\delta}: i<\kappa\right\}$.

By induction on $\xi<\lambda$ we construct a smooth $\lambda$-scale $\bar{f}=\left\langle f_{\xi}: \xi<\lambda\right\rangle \subseteq \prod_{i<\kappa} \mu_{i}$ that satisfies the following two conditions:

(1) If $\delta<\lambda$ is a limit and $\operatorname{cf} \delta \leqslant \kappa$, then $f_{\delta}(i)=\sup _{\xi \in D_{\delta}} f_{\xi}(i)$.

(2) For each $\xi<\lambda$ the set $\left[f_{\xi}, f_{\xi+1}\right)=\left\{(i, \alpha): f_{\xi}(i) \leqslant \alpha<f_{\xi+1}(i)\right\}$ has cardinality $\mu$. 
By induction on $\xi<\lambda$ we define an increasing and continuous sequence of ordinals $\zeta(\xi)<\lambda$ and a $<^{*}$-increasing sequence of functions $f_{\xi} \in \prod_{i<\kappa} \mu_{i}$ so that $f_{\xi}=g_{\zeta(\xi)}$ for all $\xi<\lambda$ except when $\xi$ is limit of cofinality $\leqslant \kappa$. Then $\bar{f}:=\left\langle f_{\xi}: \xi<\right.$ $\lambda)$ will be a smooth $\lambda$-scale as required.

At a limit stage $\xi$ of cofinality $\leqslant \kappa$, let $\zeta(\xi)=\bigcup_{\xi^{\prime}<\xi} \zeta\left(\xi^{\prime}\right)$ and use condition (1) to define $f_{\xi}$; at successor $\xi+1$ choose $\zeta(\xi+1)$ so that $\max \left\{f_{\xi}, g_{\zeta(\xi)}\right\}<^{*} g_{\zeta(\xi+1)}$ and (2) holds, and let $f_{\xi+1}=g_{\zeta(\xi+1)}$. Suppose now that $\xi$ is a limit of cofinality $>\kappa$. By the smoothness of $\bar{g}$, and since $\left\langle g_{\zeta\left(\xi^{\prime}\right)}: \xi^{\prime}<\xi\right\rangle$ is $<^{*}$-increasing, after defining $\zeta(\xi)=\bigcup_{\xi^{\prime}<\xi} \zeta\left(\xi^{\prime}\right)$ we get that $g_{\zeta(\xi)}$ is an exact upper bound of $\left\langle g_{\zeta\left(\xi^{\prime}\right)}: \xi^{\prime}<\xi\right\rangle$. But then $g_{\zeta(\xi)}$ is also an exact upper bound of $\left\langle f_{\zeta\left(\xi^{\prime}\right)}: \xi^{\prime}<\xi\right\rangle$, and we let $f_{\xi}=g_{\zeta(\xi)}$.

Let $f_{\lambda}$ be defined on $\kappa$ by $f_{\lambda}(i)=\mu_{i}$.

Claim 2.7. Suppose $\delta \leqslant \lambda$ and $A \subseteq\left[0, f_{\delta}\right)$ has cardinality $\mu$. If $\operatorname{cf} \delta>\kappa$, there is some $\delta^{\prime}<\delta$ so that $\left|A \cap\left[0, f_{\delta^{\prime}}\right)\right|=\mu$.

Proof. Find $g<f_{\delta}$ so that $\sum_{i<\kappa}|A \cap(i \times g(i))|=\mu$. By smoothness there exists some $\delta^{\prime}<\delta$ so that $g<^{*} g_{\delta^{\prime}}$.

For every $\xi<\lambda$, let $A_{\xi}=\left[f_{\xi}, f_{\xi+1}\right)$ and let $\mathcal{A}=\left\{A_{\xi}: \xi<\lambda\right\}$. Then $\mathcal{A} \subseteq$ $\mathcal{P}\left(\left[0, f_{\lambda}\right)\right)$ is $\mu$-almost disjoint and $|\mathcal{A}|=\lambda$.

For each $\delta \in S$ and $i<\kappa$, let $F_{i}^{\delta}=\left[f_{\gamma_{i}^{\delta}}, f_{\gamma_{i+1}^{\delta}}\right)$. Then $\mathcal{F}_{\delta}=\left\{F_{i}^{\delta}: i<\kappa\right\}$ is a $\mu$-almost disjoint family whose union is, by condition (1) on $\bar{f}$, equal to $\left[0, f_{\delta}\right)$. Fix a $\mu$-ad family $\mathcal{B}_{\delta} \subseteq \mathcal{P}\left(\left[0, f_{\delta}\right)\right)$ such that $\left|\mathcal{B}_{\delta}\right|=\mathfrak{a}_{\mu}, \mathcal{B}_{\delta} \cup \mathcal{F}_{\delta}$ is $\mu$-mad and $\mathcal{B}_{\delta} \cap \mathcal{F}_{\delta}=\emptyset$ (by Lemma 1.2).

Claim 2.8. If $\delta \in S$ and $B \in \mathcal{B}_{\delta}$, then for all $i<\kappa$, it follows that $\left|B \cap\left[0, f_{\gamma_{i}^{\delta}}\right)\right|<\mu$.

Proof. If not so, let $i_{0}<\kappa$ be the largest value so that $\left|B \cap\left[0, f_{\gamma_{i_{0}}^{\delta}}\right)\right|<\mu ; i_{0}$ exists because $D_{\delta}$ is closed. Now $\left|B \cap F_{i_{0}}^{\delta}\right|=\mu$, a contradiction.

Let $\mathcal{B}=\bigcup_{\delta \in S} \mathcal{B}_{\delta}$. Then $|\mathcal{B}|=\mathfrak{a}_{\mu} \cdot \lambda=\lambda$, and therefore $|\mathcal{A} \cup \mathcal{B}|=\lambda$. We will show now that $\mathcal{A} \cup \mathcal{B}$ is $\mu$-mad.

Suppose that $A=A_{\xi} \in \mathcal{A}$ and $B \in \mathcal{B}_{\delta}$ for some $\delta \in S$. If $\xi \geqslant \delta$, then clearly $|A \cap B|<\mu$, and if $\xi<\delta$, there is some $i<\kappa$ so that $A_{\xi} \subseteq^{*} F_{i}^{\delta}$ and $|A \cap B|<\mu$ follows from Claim 2.8 .

If $B_{1} \in \mathcal{B}_{\delta_{1}}$ and $B_{2} \in \mathcal{B}_{\delta_{2}}$ with $\delta_{1}<\delta_{2}$ in $S$, then there is some $i<\kappa$ so that $f_{\delta_{1}}<f_{\gamma_{i}^{\delta_{2}}}$ and Claim 2.8 gives $\left|B_{1} \cap B_{2}\right|<\mu$.

This establishes that $\mathcal{A} \cup \mathcal{B}$ is $\mu$-mad. To verify maximality, let $Z \subseteq\left[0, f_{\lambda}\right)$ be arbitrary of size $\mu$. By Claim 2.7 the first $\xi \leqslant \lambda$ for which $\left|Z \cap\left[0, f_{\xi}\right)\right|=\mu$ is either a successor or of cofinality $\leqslant \kappa$. Cofinality $<\kappa$ is ruled out by condition (1) on $\bar{f}$. The case $\xi$ successor implies that $\left|Z \cap A_{\xi}\right|=\mu$. Finally, in the remaining case $\xi=\delta \in S$, there is some $B \in \mathcal{B}_{\delta}$ so that $|Z \cap B|=\mu$.

Now the proof of Theorem 2.3 will be completed by the following lemma, whose proof is actually found implicitly in [15]. We shall sketch a proof here too.

Lemma 2.9. Suppose $\mu$ is singular and $\mu<\lambda<\mathfrak{b}_{\mu}$, $\lambda$ regular. Then there is a smooth $(\mu, \lambda)$-scale. If $\mathfrak{b}_{\mu}$ is a successor of a regular cardinal, there is also a smooth $\left(\mu, \mathfrak{b}_{\mu}\right)$-scale.

Proof. Since $\lambda<\mathfrak{b}_{\mu}$, there exists a product $\prod_{i<\kappa} \mu_{i}$, where $\kappa=\operatorname{cf} \mu$, so that $\mathfrak{b}\left(\prod_{i<\kappa} \mu_{i},<^{*}\right)>\lambda$. 
By Claim 1.3 in [15] there exists a $\lambda$-scale $\bar{f}=\left\langle f_{\alpha}: \alpha<\lambda\right\rangle$ in some $\prod_{i<\kappa} \mu_{i}^{\prime}$ such that for all regular $\theta \in(\kappa, \mu)$, every $\alpha<\lambda$ with $\operatorname{cf} \alpha=\theta$ satisfies that $\bar{f} \uparrow \alpha$ is flat, that is, is equivalent modulo the bounded ideal on $\kappa$ to a strictly increasing sequence of ordinal functions on $\kappa$.

By Lemma 15 in [10], every $\alpha<\lambda$ with cf $\alpha>\kappa$ satisfies that $\bar{f} \uparrow \alpha$ has an exact upper bound. Now it is clear how to replace $\bar{f}$ by a smooth $\lambda$-scale.

Suppose now that $\mathfrak{b}_{\mu}=\lambda^{+}, \lambda=\operatorname{cf} \lambda$. By [14, 4.1, the set $S_{<\lambda}^{\lambda^{+}}:=\{\alpha: \alpha<$ $\left.\lambda^{+} \wedge \mathrm{cf} \alpha<\lambda\right\}$ is a union of $\lambda$ sets, each of which carries a square sequence. Therefore, $S_{<\lambda}^{\lambda^{+}} \in I[\lambda]$. By 2.5 in chapter 1 of [15], there exists a $\left(\mu, \mathfrak{b}_{\mu}\right)$-scale in which all points of cofinality $<\mu$ are flat and therefore a smooth $\left(\mu, \mathfrak{b}_{\mu}\right)$-scale.

In contrast to the case of singular $\mu$, let us mention the following result of $\mathrm{A}$. Blass 4, which generalizes Hechler's result [8]: it is consistent that $\operatorname{MAD}\left(\aleph_{0}\right)=$ $C$, for any prescribed closed set of uncountable cardinals $C$ that satisfies that $\left[\aleph_{1}, \aleph_{1}+|C|\right] \subseteq C$ and $\lambda^{+} \in C$ whenever $\lambda \in C$ has countable cofinality. For example, by Blass' or by Hechler's results there are universes of set theory in which $\operatorname{MAD}\left(\aleph_{0}\right)=\left\{\aleph_{1}, \aleph_{\omega+1}\right\}$. By Corollary 2.5, in any universe that satisfies this, it follows that $\left[\aleph_{1}, \aleph_{\omega+1}\right] \subseteq \operatorname{MAD}\left(\aleph_{\omega}\right)$.

Recently Brendle [5], using techniques from [16], proved the consistency of $\mathfrak{a}=$ $\aleph_{\omega}$.

Problem 2.10. Is it consistent that $\mathfrak{a}_{\aleph_{\omega}}=\aleph_{\omega}$ ?

\section{Consistency Results on MAD $\left(\aleph_{\omega}\right)$ From large CARDinal AxiOms}

The inequality (1) can be used to control $\operatorname{MAD}\left(\aleph_{\omega}\right)$ by first increasing $\mathfrak{b}_{\aleph_{\omega}}$ and then increasing $\mathfrak{b}$. PCF theory implies that whenever the SCH fails at a singular cardinal $\mu$, it follows that $\mathfrak{b}_{\mu}>\mu^{+}$. On the other hand, $\mathfrak{b}_{\mu}$ cannot be changed by a ccc forcing.

Before we state the result, let us recall some pcf terminology:

$$
\operatorname{pcf}\left\{\aleph_{n}: n<\omega\right\}=\left\{\mathfrak{b}\left(\prod_{n} \aleph_{n}, \leqslant_{I}\right): I \subseteq \mathcal{P}(\omega) \text { is a proper ideal }\right\} .
$$

The relation $<_{I}$ is defined by $f<_{I} g \Leftrightarrow\{n: f(n) \geqslant g(n)\} \in I$.

$\operatorname{pcf}\left\{\aleph_{n}: n<\omega\right\}$ is an interval of regular cardinals and has a maximum. For every $\lambda \in \operatorname{pcf}\left\{\aleph_{n}: n<\omega\right\}$ there exists a pcf generator $B_{\lambda} \subseteq \omega$ so that the following holds: denote by $J_{<\lambda}$ the ideal that is generated by $\left\{B_{\theta}: \theta \in \operatorname{pcf}\left\{\aleph_{n}: n<\omega\right\} \wedge \theta<\lambda\right\}$; then

$$
\lambda=\mathfrak{b}\left(\prod_{n} \aleph_{n}, \leqslant_{<\lambda}\right) .
$$

Finally, $\left(\aleph_{\omega}\right)^{\aleph_{0}}=\max \operatorname{pcf}\left\{\aleph_{n}: n<\omega\right\} \times 2^{\aleph_{0}}$. Therefore, if $\aleph_{\omega}$ is a strong limit, $2^{\aleph_{\omega}}=\max \operatorname{pcf}\left\{\aleph_{n}: n<\omega\right\}$.

Fact 3.1. For every $\beta<\omega_{1}$ it is consistent (from large cardinal axioms) that $2^{\aleph_{\omega}}=$ $\mathfrak{b}_{\mu}=\aleph_{\omega+\beta+1}$.

Proof. Let $V$ be any universe of set theory in which $\aleph_{\omega}$ is a strong limit cardinal and $2^{\aleph_{\omega}}=\max \operatorname{pcf}\left\{\aleph_{n}: n \in \omega\right\}=\aleph_{\omega+\beta+1}$ [13], 9].

In $V$, the ideal $J_{<\max \operatorname{pcf}\left\{\aleph_{n}: n<\omega\right\}}$ is proper and is generated by countably many sets. Therefore, by simple diagonalization there exists an infinite $B \subseteq \omega$ so that 
$J_{<\operatorname{maxpcf}\left\{\aleph_{n}: n<\omega\right\}} \mid B$ is contained in the ideal of finite subsets of $B$. Since $\mathfrak{b}\left(\prod_{n} \aleph_{n}, \leqslant J_{<\operatorname{maxpcf}\left\{\aleph_{n}: n<\omega\right\}}\right)=\aleph_{\omega+\beta+1}$, it follows that $\mathfrak{b}\left(\prod_{n \in B} \aleph_{n}, \leqslant^{*}\right)=\aleph_{\omega+\beta+1}$; hence $\mathfrak{b}_{\aleph_{\omega}}=\aleph_{\omega+\beta+1}$.

Theorem 3.2. For every $\beta<\omega_{1}$ and $\alpha \leqslant \omega+\beta+2$, it is consistent (from large cardinals) that $2^{\aleph_{\omega}}=\aleph_{\omega+\beta+2}$ and $\operatorname{MAD}\left(\aleph_{\omega}\right)=\left[\aleph_{\alpha}, \aleph_{\omega+\beta+2}\right]$.

Proof. Start from a model $V$ in which $2^{\aleph_{0}}=\aleph_{1}$, $\aleph_{\omega}$ is strong limit and $2^{\aleph_{\omega}}=$ $\aleph_{\omega+\beta+2}$. Such a model exists by the previous Fact.

For every regular $\aleph_{\omega}<\lambda \leqslant \aleph_{\omega+\beta+2}$, there is a smooth $\lambda$-scale by Lemma 2.9 Consequently, there is also a smooth $\aleph_{\omega+\beta+2}$-scale.

Now apply Theorem 2.3 to finish the proof.

By Theorem 5.4(b) in [3], after adding many Cohen subsets to $\omega_{1}, \max \operatorname{MAD}\left(\aleph_{\omega}\right)$ does not increase by much. Therefore, it is consistent to have $\operatorname{MAD}\left(\aleph_{\omega}\right)=\left[\aleph_{1}\right.$, $\left.\aleph_{\omega+\beta+2}\right]$ as above, and to have $2^{\aleph_{\omega}}$ arbitrarily large.

\section{ACKNOWLEDGMENTS}

The second author would like to thank Uri Abraham for fruitful discussions of some of the proofs in this paper and to thank Isaac Gorelic for useful remarks.

\section{REFERENCES}

[1] B. Balcar and P. Simon. On collections of almost disjoint families, Comment. Math. Univ. Carolinae 29 (1988), no 4, pp. 631-646. MR 90b:03072

[2] B. Balcar and P. Simon. Disjoint refinements, in: Handbook of Boolean Algebras, eds. J. D. Monk, R. Bonnet, vol. 2. North-Holland, Amsterdam, 1989.

[3] J. E. Baumgartner. Almost-disjoint sets, the dense set problem and the partition calculus, Ann. Math. Logic 9 (1976), no. 4, 401-439. MR 53:5299

[4] A. Blass, Simple cardinal characteristics of the continuum, in: Set theory of the reals, Israel Math. Conf. Proc. 6, Bar-Ilan Univ., Ramat Gan, 1993. (1991) 63-90. MR 94i:03098

[5] J. Brendle. The almost disjointness number may have countable cofinality, Trans. Amer. Math. Soc. 355 (2003), no. 7, 2633-2649 (electronic). MR 2004c:03062

[6] E. K. van Douwen, The integers and topology, in: Handbook of set-theoretic topology, 111-167, North-Holland, Amsterdam, 1984. MR 87f:54008

[7] P. ERdös, S. Hechler, On maximal almost-disjoint families over singular cardinals, Colloquia Mathematica Societatis János Bolyai 10, Infinite and finite sets, Keszthely (Hungary) 1973, pp. 597-604. MR 51:12530

[8] S. Hechler. Short complete nested sequences in $\beta \mathbb{N}-\mathbb{N}$ and small maximal almost disjoint families, Gen. Top. Appl. 2 (1972), 139-149. MR 46:7028

[9] M. Gitik and M. Magidor. The singular cardinal hypothesis revisited, Set theory of the continuum (Berkeley, CA, 1989), 243-279, Math. Sci. Res. Inst. Publ., 26, Springer, New York, 1992. MR 95c:03131

[10] M. Kojman. Exact upper bounds and their uses in set theory, Ann. Pure Appl. Logic 92 (1998), no. 3, 267-282. MR 2000b:03163

[11] E. Milner, K. Prikry, Almost disjoint sets, in: Surveys in combinatorics, (New Cross 1987) pp. 155-172, Cambridge, 1987. MR 88k:04001

[12] J. D. Monk, The spectrum of partitions of a Boolean algebra, Arch. Math. Logic 40 (2001), no. 4, 243-254. MR 2002j:03073

[13] S. Shelah, The singular cardinals problem: independence results., Surveys in set theory, 116-134, London Math. Soc. Lecture Note Ser., 87, Cambridge Univ. Press, Cambridge, 1983. MR 87b:03114

[14] S. Shelah. Reflecting stationary sets and successors of singular cardinals, Arch. Math. Logic 31 (1991), no. 1, 25-53. MR 93h:03072 
[15] S. Shelah. Cardinal Arithmetic, Oxford University Press, 1994. MR 96e:03001

[16] S. Shelah. Are $\mathfrak{a}$ and $\mathfrak{d}$ your cup of tea?, Acta Math., to appear.

Department of Mathematics, Ben-Gurion University of the Negev, Beer-Sheva, IsRAEL

E-mail address: kojman@math.bgu.ac.il

Department of Mathematics, Ben-Gurion University of the Negev, Beer-Sheva, IsRael - And - Institute of Mathematics, University of Silesia, Katowice, Poland

E-mail address: kubis@math.bgu.ac.il

Institute of Mathematics, Hebrew University of Jerusalem, IsRael - ANd - Department of Mathematics, Rutgers University, New Brunswick, New Jersey

E-mail address: shelah@math.huji.ac.il 